\title{
High-Energy Hadron-Nucleus Scattering and the Composite Structure of Nucleons.
}

S. FORTE

Istituto di Fisica Teorica dell'Università - Torino, Italia

E. Predazzi

Istituto di Fisica Teorica dell'Università - Torino, Italia

Istituto Nazionale di Fisica Nucleare - Sezione di Torino, Italia

(Nuovo Cimento $A, \mathbf{8 8}, 391$ (1985))

Please note that the following two corrections should be made in this paper.

On page 394, in the second of eqs. (4) a multiplication point should substitute the comma at the end of the first line.

On page 398 , the term in square brackets of eq. (14) should read

$$
\left[-q^{2} \frac{A-a\left(l_{1}, \ldots, l_{\Lambda}\right) r_{A}^{2}}{4 A a\left(l_{1}, \ldots, l_{\Lambda}\right)}\right] \text {. }
$$

(C) by Socleta Italiana di Fisica

Proprietà letteraria riservata

Direttore responsablle: RENATO ANGELO RICCI

Stampato in Bologna dalla Tipografia Compositori oot tipi della Tipografla Monograf
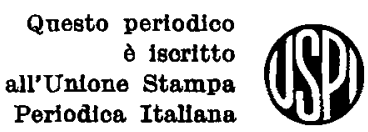

Questo fascicolo è stato licenziato dai torchi il 21-IV-1986 